Brazilian Journal

of Chemical

ISSN 0104-6632

Engineering

\title{
PREPARATION AND CHARACTERIZATION OF IMMOBILIZED SPORES WITH LACCASE ACTIVITY FROM Bacillus pumilus W3 ON DEAE-CELLULOSE AND THEIR APPLICATION IN DYE DECOLORIZATION
}

\author{
Wen Zhou, Zheng-Bing Guan*, Yu-Jie Cai, Yu Chen, Ning Zhang and Xiang-Ru Liao* \\ The Key Laboratory of Industrial Biotechnology, Ministry of Education, \\ School of Biotechnology, Jiangnan University, Wuxi 214122, China. \\ Phone: + 865108532 7725; Fax: + 8651085327725 \\ E-mail: guanzb525@163.com; liaoxiangru@163.com
}

(Submitted: August 5, 2015 ; Revised: November 10, 2015 ; Accepted: November 24, 2015)

\begin{abstract}
In order to obtain a more stable and reusable immobilized spore laccase for dye decolorization, the spores from Bacillus pumilus W3 were immobilized on diethylaminoethyl cellulose (DEAE-cellulose). Free and immobilized spore laccase retained $34.38 \%$ and $46.11 \%$ of their initial activity, respectively, after 10 days incubation at $\mathrm{pH} 9.0$. Their residual activity remained at $27.36 \%$ and $31.84 \%$ after $10 \mathrm{~h}$ incubation at $70{ }^{\circ} \mathrm{C}$. Immobilized spore laccase was more stable than free spore laccase in the presence of most organic solvents, metal ions and inhibitors. The tested dyes, including methyl green, methyl red and acid red 1 , were removed $86.82 \%, 78.14 \%$ and $88.60 \%$, respectively, by immobilized spore laccase after $24 \mathrm{~h}$ at $37{ }^{\circ} \mathrm{C}$, and $74.34 \%$ of initial decolorization activity after 7 cycles was retained when it decolorized acid red 1 . These properties indicated that immobilized spore laccase may be useful in textile effluent treatment.

Keywords: Spore laccase; Bacillus pumilus; DEAE-cellulose; Decolorization; Immobilization.
\end{abstract}

\section{INTRODUCTION}

Synthetic dyes are used extensively in textile dyeing and finishing industries. More than $7.0 \times 10^{5}$ tons and $1.0 \times 10^{5}$ kinds of commercially available dyes are produced worldwide annually (Strong and Claus 2011). Many of the synthetic dyes are toxic, carcinogenic, mutagenic, or teratogenic to various aquatic organisms and are difficult to degrade (Ozmen et al., 2008). Growing demand of dyes in the textile industry makes it one of the main sources of water pollution problems (Sarayu and Sandhya 2012).

In early years, some physical and chemical methods, such as adsorption, oxidation, coagulation-floc- culation, filtration, and electrochemical methods were the main treatment methods. However, these methods are quite expensive and have some operational problems (Madhavi and Lele 2009). Bacterial aerobic or anaerobic dye degradation is one of the treatment methods for dye-containing industrial effluent. However, bacterial aerobic dye degradation has been confined to chemostat-enriched cultures adapting to a single dye, while under anaerobic conditions the azo dyes are cleaved by azo-reductases to yield potentially carcinogenic aromatic amines (Strong and Claus 2011).

Laccase (EC 1.10.3.2), one of the earliest discovered multicopper oxidases, is able to decolorize

*To whom correspondence should be addressed 
synthetic dyes efficiently, which attracted a large number of scholars to apply it in dye decolorization. To date, white-rot fungi are the most efficient single class of microorganisms in breaking down synthetic dyes (Wesenberg et al., 2003). However, fungal laccase is usually unstable under high temperatures and alkaline conditions, and no decolorization activities are observed at $\mathrm{pH}$ values higher than 7 (Held et al., 2005). In contrast, bacterial laccase can withstand high temperature and extreme $\mathrm{pH}$ values (Zhang et al., 2012).

Spore laccase is a typical bacterial laccase. It was mentioned by Held et al (2005) for the first time and may be defined as spore-bound laccase (laccase is one of the spore proteins) with the individual spore as the research object. CotA, an outer coat protein of spores from the Bacillus genus, is the best-studied bacterial laccase by far and usually considered as the laccase activity supplier of spore laccase. However, there may be some other spore proteins with laccase activity such as MnxG from the ridged outermost layer of the SG-1 spores (van Waasbergen et al., 1996). The properties of spore laccase from previous reports (Lončar et al., 2014; Lu et al., 2012b) have shown their advantages for the biodegradation of industrial textile dyes.

The immobilization of laccase offers several improvements for dye decolorization because the stability of laccase towards $\mathrm{pH}$, temperature and storage is frequently enhanced. Moreover, the reusability of immobilized laccase represents a great advantage compared with free laccase (Fernández-Fernández et al., 2013). The immobilization of fungal laccase on different carriers by various means for dye decolorization has been well investigated, such as green coconut fiber via covalent attachment (Cristóvão et al., 2012), $\mathrm{Fe}_{3} \mathrm{O}_{4} / \mathrm{SiO}_{2}$ nanoparticles with particle size below $30 \mathrm{~nm}$ by immobilization through glutaraldehyde coupling (Wang et al., 2013), alginate-gelatin mixed gel via entrapment (Mogharabi et al., 2012), beer spent grain via adsorption and covalent binding (da Silva et al., 2012), etc. However, immobilized fungal laccase still prefers acidic conditions (Bayramoglu et al., 2012). To date, there are only a few reports (Held et al., 2005; Lu et al., 2012b) about spore laccase immobilization. These reports showed the advantages of immobilized spore laccase in dye decolorization. However, the optimization of immobilization conditions of spore laccase, the characterization of immobilized spore laccase and its application in dye decolorization still need to be studied further in detail.

In the present work, we describe, for the first time, the immobilization of spores from Bacillus pumilus W3 on diethylaminoethyl cellulose (DEAEcellulose). The decolorization ability of free and immobilized spore laccases was determined by using various synthetic dyes. Furthermore, the characterizations of free and immobilized spore laccases were also assessed in comparison.

\section{MATERIALS AND METHODS}

\section{Materials and Bacterial Strain}

2,2'-Azino-bis(3-ethylbenzothiazoline-6-sulfonate) (ABTS), syringaldazine (SGZ), 2,6-dimethoxyphenol (2,6-DMP), syringaldehyde (SYR), 1-hydroxybenzotriazole (HBT), vanillin, and acid red 1 were all of reagent grade and obtained from Sigma-Aldrich (St. Louis, MO, USA). DEAE-cellulose (cotton fiber), 4hydroxybenzoic acid (4-HBA), crystal violet, methyl green, methyl red, lysozyme (twice crystallized), phenylmethylsulfonyl fluoride (PMSF) and other chemicals were all of analytical grade and purchased from Sinopharm Chemical Reagent Company (Shanghai, China). B. pumilus W3 was isolated from raw gallnut honey samples (Guan et al., 2014a), and deposited at $-20{ }^{\circ} \mathrm{C}$.

\section{Preparation of Spore Suspension}

B. pumilus $\mathrm{W} 3$ was cultivated on nutrient broth sporulation medium containing $0.2 \mathrm{mM} \mathrm{Cu}^{2+}$ at $30^{\circ} \mathrm{C}$, $200 \mathrm{rpm}$ for $48 \mathrm{~h}$ (Schaeffer et al., 1965). Spore suspension was prepared following a procedure published by Jenkinson et al. (1981) with some modifications. The culture fluid was harvested by centrifugation $\left(8000 \mathrm{rpm}, 10 \mathrm{~min}, 4{ }^{\circ} \mathrm{C}\right)$ and washing twice with deionized water, then suspended in deionized water containing lysozyme $(1 \mathrm{mg} / \mathrm{ml})$ at $37^{\circ} \mathrm{C}$ for $2 \mathrm{~h}$ to lyse remaining vegetative cells. The pellets were washed with $\mathrm{NaCl}(1 \mathrm{M})$ and $\mathrm{KCl}(1 \mathrm{M})$, and finally suspended in deionized water to a concentration of $10 \mathrm{mg}$ wet spores $/ \mathrm{ml}$. The purified spores were stored at $4{ }^{\circ} \mathrm{C}$.

\section{Enzyme Assay}

Laccase activity, for both free and immobilized spore laccases, was assayed at $37{ }^{\circ} \mathrm{C}$ using ABTS (0.5 mM), SGZ (0.05 mM), and 2,6-DMP (1.5 mM) as substrates. The oxidation of ABTS was measured at $420 \mathrm{~nm}$, of SGZ at $525 \mathrm{~nm}$ and of 2,6-DMP at 469 $\mathrm{nm}$ in $0.1 \mathrm{M}$ citrate-phosphate buffer $(\mathrm{pH} 3.0-7.0)$ or $0.1 \mathrm{M}$ Tris- $\mathrm{HCl}$ buffer (pH 7.0-9.0). Reactions started with the addition of substrates. The oxidation 
of substrates was monitored using an UV-Visible spectrophotometer (METASH, UV-6000). One unit of enzyme activity was defined as the amount of enzyme required to oxidize $1 \mu \mathrm{mol}$ of substrate per minute. All assays were carried out in triplicate.

\section{Immobilization of Spore Laccase}

DEAE-cellulose was pretreated according to the previous report (Liu et al., 2014). Briefly, dry DEAEcellulose was suspended in 5 volumes of distilled water and incubated overnight after stirring for swelling and mixing of the slurry. The swelled DEAEcellulose was filtered on a Buchner funnel and then incubated with $0.5 \mathrm{M} \mathrm{HCl}$ for $1 \mathrm{~h}$. It was then washed with distilled water until neutral $\mathrm{pH}$. It was further incubated with $0.5 \mathrm{M} \mathrm{NaOH}$ for $1 \mathrm{~h}$, and then washed with distilled water until neutral pH. $0.5 \mathrm{ml}$ of spore suspension (100 mg wet weight per $\mathrm{ml}$ ) was added to $19.5 \mathrm{ml}$ of $0.05 \mathrm{M}$ citrate-phosphate buffer and thoroughly mixed with the activated DEAEcellulose at the ratio of $1 \mathrm{ml}: 2 \mathrm{~g}$ (enzyme: DEAEcellulose dry weight). The mixture was constantly rotated for full adsorption at $37^{\circ} \mathrm{C}, 200 \mathrm{rpm}$ overnight. The mixture in full adsorption was washed three times with $0.1 \mathrm{M}$ citrate-phosphate buffer $(\mathrm{pH}$ 6.8) and filtered on a Buchner funnel. The filtrate was collected and used for evaluating the immobilization efficiency. The laccase activity of immobilized spores was measured. The immobilization yield was defined as previously described by Valerio et al. (2013).

The effects of several factors, including solution $\mathrm{pH}$, spore amount and adsorption time, on immobilization efficiency were investigated, respectively. Scanning electron microscopy (SEM) of DEAEcellulose before and after spore immobilization was performed with a scanning electron microscope ((FEI Quanta-200, The Netherlands)) at $5.0 \mathrm{kV}$. All the experiments were performed in triplicate.

\section{Characterization of Free and Immobilized Spore Laccase Activity}

The effects of temperature and $\mathrm{pH}$ on free and immobilized spore laccase activity and stability were investigated as previously described (Guan et al., 2014b) with some modifications. The optimum $\mathrm{pH}$ was measured using ABTS, SGZ and 2,6-DMP as substrates at $37^{\circ} \mathrm{C}$ in $0.1 \mathrm{M}$ citrate-phosphate buffer ( $\mathrm{pH} 3.0-7.0)$ or $0.1 \mathrm{M}$ Tris-HCl buffer ( $\mathrm{pH}$ 7.0-9.0). The $\mathrm{pH}$ stability of free and immobilized spore laccases was assayed by pre-incubating the enzyme at $\mathrm{pH} 3.0,7.0$, and 9.0 at $37^{\circ} \mathrm{C}$ for 10 days. The opti- mum temperature for laccase activity was measured using SGZ as the substrate at different temperatures $\left(25-90{ }^{\circ} \mathrm{C}\right)$ at the optimum $\mathrm{pH}$ condition. For thermal stability analysis, the enzyme was incubated at different temperatures $\left(60,70\right.$ and $\left.80{ }^{\circ} \mathrm{C}\right)$ for $0 \mathrm{~h}$ to $10 \mathrm{~h}$, and then incubated in ice water for $10 \mathrm{~min}$, and the residual activity was measured.

The effects of organic solvents on laccase activity were investigated in the presence of $10 \%(\mathrm{v} / \mathrm{v})$ and $50 \%(\mathrm{v} / \mathrm{v})$ organic solvents. Free and immobilized spores were pre-incubated in $0.1 \mathrm{M}$ citrate-phosphate buffer ( $\mathrm{pH} 6.8$ ) at $37^{\circ} \mathrm{C}$ for $2 \mathrm{~h}$, and then the residual activity was determined. The effects of metal ions on the enzyme activity were investigated in the presence of $10 \mathrm{mM}$ of $\mathrm{K}^{+}, \mathrm{Ca}^{2+}, \mathrm{Na}^{+}, \mathrm{Mg}^{2+}, \mathrm{Al}^{3+}, \mathrm{Mn}^{2+}, \mathrm{Fe}^{3+}$, $\mathrm{Co}^{2+}, \mathrm{Ni}^{2+}, \mathrm{Cu}^{2+}$, and $\mathrm{Zn}^{2+}$. To study the effects of inhibitors on enzyme activity, free and immobilized spores were pre-incubated with various inhibitors at different concentrations at $37^{\circ} \mathrm{C}$ for $15 \mathrm{~min}$, and then the residual activities were determined. All the experiments were performed in triplicate at the optimum $\mathrm{pH}$ condition. The samples in the absence of organic solvents, inhibitors and metal ions were used as the control, and were run in parallel.

\section{Dye Decolorization}

Dye decolorization experiments were carried out using methyl red, acid red 1 and methyl green. The reaction mixture $(4 \mathrm{~mL})$ contained reaction buffer, $0.1 \mathrm{ml}$ mediator $(4 \mathrm{mM}), 0.1 \mathrm{~mL}$ dyes $(4 \mathrm{~g} / \mathrm{L})$ and appropriate quantities of spores or immobilized spores with the same weight as free spores. The free spores acted as the catalyst to find the most suitable mediator, a variety of compounds, including HBT, ABTS, SYR, vanillin, and 4-HBA, were added to the reaction mixture, respectively, and the best promotion effect on dye decolorization was selected. The optimum $\mathrm{pH}$, spore amount, dye concentration and treatment time for dye decolorization were investigated using the free spores as the catalyst. The maximal absorbance wavelength of several dyes and the decolorization ability of enzyme was determined by spectral scanning and absorbance detection using an UV-Visible spectrophotometer (METASH, UV-6000), respectively. The samples without the addition of enzyme, as the control, were run in parallel, and all samples were incubated and rotated at $37^{\circ} \mathrm{C}, 200 \mathrm{rpm}$ for $24 \mathrm{~h}$. Decolorization percentage was recorded as $\left(A_{c}-A_{e}\right) / A_{c} * 100 \%$, where $A_{c}$ and $A_{e}$ are the control and experimental group, respectively, which is more reasonable than calculating the decrease of absorbance relative to the initial absorbance because lots of dyes decolorize automatically. 
The reusability of immobilized spore laccase was evaluated by measuring the percentage decolorization of dyes by immobilized spore laccase after seven cycles. The immobilized spores were separated from the reaction mixture through filtration and washed three times for reuse. The measuring method was the same as the method described above. All the experiments were performed in triplicate.

\section{RESULTS}

\section{Immobilization of Spore Laccase}

\section{Effect of pH on Immobilization Efficiency}

The effect of $\mathrm{pH}$ on immobilization efficiency of spore laccase was investigated in the range of $\mathrm{pH}$ 5.0-10.5. As shown in Figure 1, the immobilization yield of spores always remained above $80 \%$, which increased with increasing $\mathrm{pH}$ and reached a maximum $(99.19 \%)$ at about $\mathrm{pH} 10.0$. The laccase activity of immobilized spores also increased with increasing $\mathrm{pH}$ and reached the highest value, $3.01 \mathrm{U} / \mathrm{g}$ wet spores, at $\mathrm{pH} 10.0$.

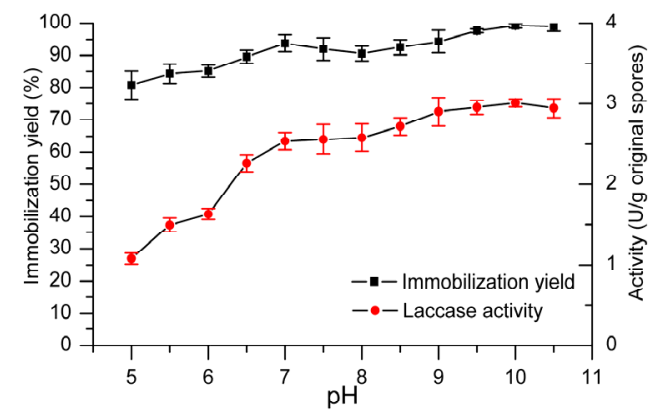

Figure 1: Effect of $\mathrm{pH}$ on immobilization efficiency of spore laccase. Adsorption buffer: $0.05 \mathrm{M}$ citratephosphate buffer for $\mathrm{pH} 5.0-7.0,0.05 \mathrm{M}$ Tris- $\mathrm{HCl}$ buffer for $\mathrm{pH} 7.5-9.0$ and $0.05 \mathrm{M}$ sodium carbonatesodium bicarbonate buffer for $\mathrm{pH}$ 9.5-10.5.

\section{Effect of Spore Amount and Adsorption Time on Immobilized Efficiency}

The effect of spore amount on immobilization efficiency was investigated ranging from 10 to $150 \mathrm{mg}$ wet spores per g dry weight DEAE-cellulose (Figure A1a). The relative activity of immobilized spore laccase increased rapidly with the increase of spore amount when the spore concentration was $10 \mathrm{mg} / \mathrm{g}$ to $50 \mathrm{mg} / \mathrm{g}$, and increased slowly when the spore concentration exceeded $50 \mathrm{mg} / \mathrm{g}$. Nonetheless, only a trace amount of enzyme activity was found in the supernatant (about $0.2 \%-5 \%$ of the total activity). The relative activity increased with prolonged adsorption time from 0 to $24 \mathrm{~h}$ (Figure A1b). The relative activity of immobilized spore laccase arrived at $98 \%$ at $5 \mathrm{~h}$, and there was no laccase activity increase after $10 \mathrm{~h}$.
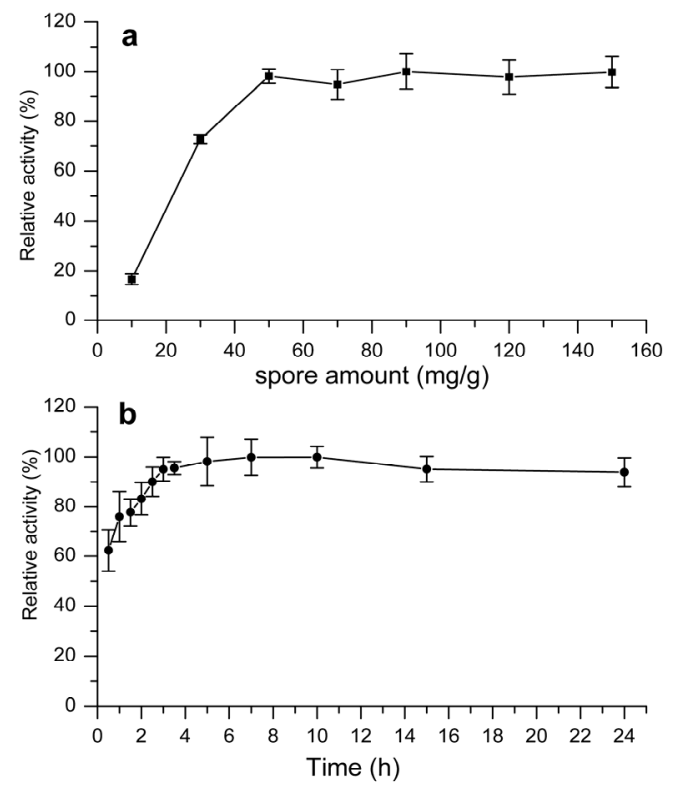

Figure A1: Effects of spore amount (a) and absorption time (b) on immobilization efficiency. The maximum activity was set as $100 \%$. The unit "mg/g" means mg wet spores per g dry DEAEcellulose in (b).

\section{Structure Characterization of Immobilized Spores}

SEM images of the DEAE-cellulose before and after spore immobilization are shown in Figure 2. The images show the diameter of the fiber to be, on average, about $14-21 \mu \mathrm{m}$, and the spores to be, on average, about 1.0-1.2 $\mu \mathrm{m}$ long and 0.4-0.6 $\mu \mathrm{m}$ wide. Spore distribution on the fibers surface is not uniform (Figure $2 \mathrm{c}$ to $2 \mathrm{e}$ ). After a period of preservation and drying, some spores seem to blend into the fibers, and form a relief-like morphology (Figure 3f).

\section{Characterizations of Free and Immobilized Spore Laccases}

\section{Effect of pH on the Activity and Stability of Free and Immobilized Spore Laccase}

The effect of $\mathrm{pH}$ on free and immobilized spore laccases is shown in Figure 4. Both free and immobilized spore laccases demonstrated that there is a broad $\mathrm{pH}$ range for catalyzing substrates (Figure 3a). The 
optimum $\mathrm{pH}$ of free and immobilized spore laccases for oxidizing ABTS was $\mathrm{pH} 3.2$ and $\mathrm{pH} 3.4$, respectively, for oxidizing SGZ was 6.8 and 7.0, respectively, and for oxidizing 2,6-DMP was 7.0 (both of them). The $\mathrm{pH}$ stability of free and immobilized spore laccases is shown in Figure $3 \mathrm{~b}$. The residual activity of immobilized spore laccase respectively retained $52.46 \%$ and $46.11 \%$ of its initial activity at $\mathrm{pH} 7.0$ and 9.0 after 10-day incubation, while free spore laccase retained $37.51 \%$ and $34.38 \%$ of its initial activity under the same conditions. However, both free and immobilized spore laccases are unstable at $\mathrm{pH} 3.0$, and retained $17.25 \%$ and $29.73 \%$ of their initial activity, respectively, after 10 days.
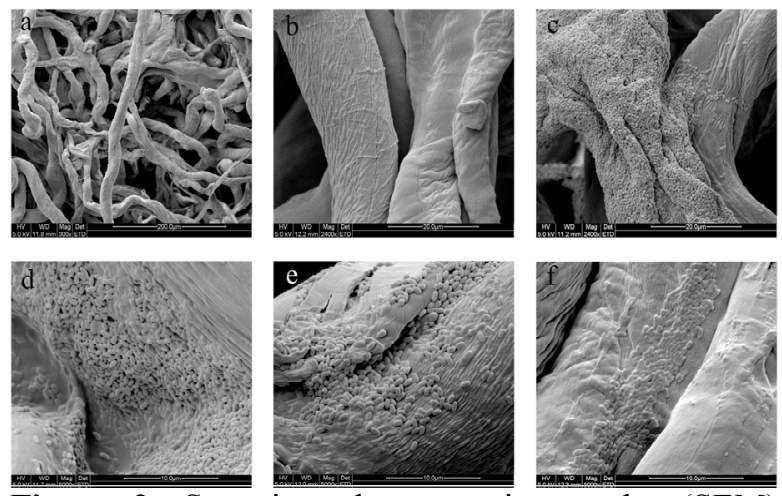

Figure 2: Scanning electron micrography (SEM) showing the surface morphologies of DEAE-cellulose before $(a, b)$ and after ( $c$ to f) spore immobilization.

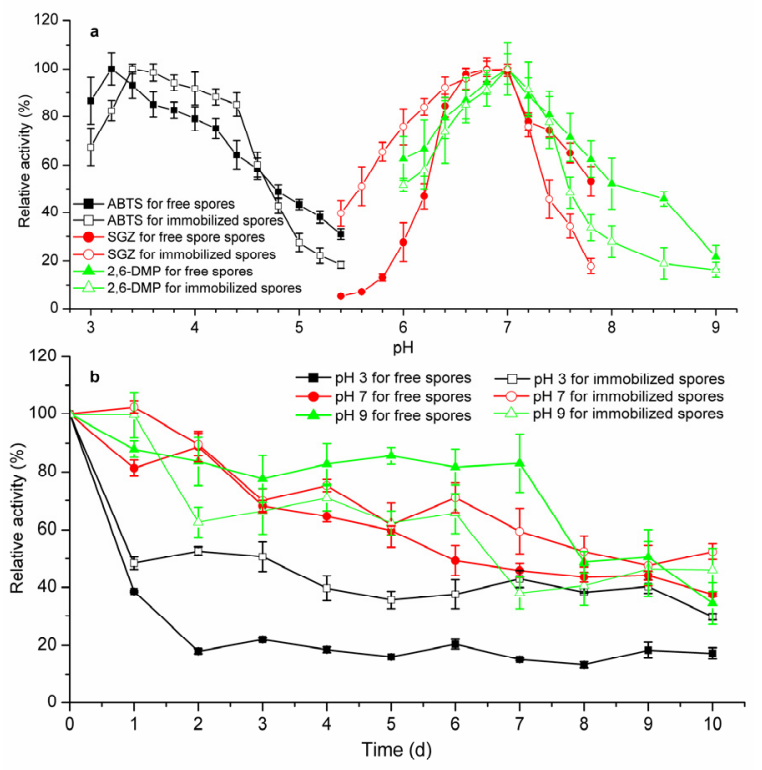

Figure 3: Effect of $\mathrm{pH}$ on free and immobilized spore laccase activity (a) and stability (b) at $37^{\circ} \mathrm{C}$. (a) ABTS, pH 3.0-5.4; SGZ, pH 5.4-7.8; 2,6-DMP, pH 6.0-9.0; (b) substrate: $\mathrm{pH} 3.0$, ABTS; $\mathrm{pH}$ 7.0, SGZ and 2,6DMP.
Effect of Temperature on the Activity and Stability of Free and Immobilized Spore Laccase

The effect of temperature on free and immobilized spore laccases towards SGZ is shown in Figure 4. The optimum temperature of immobilized spore laccase is $10^{\circ} \mathrm{C}$ lower than that of free spore laccase, and are $55^{\circ} \mathrm{C}$ and $65^{\circ} \mathrm{C}$, respectively (Figure $4 \mathrm{a}$ ). Both free and immobilized spore laccases were very stable at $60{ }^{\circ} \mathrm{C}$ after $10 \mathrm{~h}$ and retained $85.57 \%$ and $73.75 \%$ respectively, of their initial activity (Figure 4b). The half-life of free and immobilized spore laccases was about $5.5 \mathrm{~h}$ and $4.5 \mathrm{~h}$ at $70{ }^{\circ} \mathrm{C}, 2.2 \mathrm{~h}$ and $2.5 \mathrm{~h}$ at $80{ }^{\circ} \mathrm{C}$, respectively, and retained $27.36 \%$ and $31.84 \%$ of their initial activity after $10 \mathrm{~h}$ at $70{ }^{\circ} \mathrm{C}$, respectively. Immobilized spore laccase lost all activity after $10 \mathrm{~h}$ at $80^{\circ} \mathrm{C}$ while free spore laccase lost all activity after $8 \mathrm{~h}$ at $80^{\circ} \mathrm{C}$.
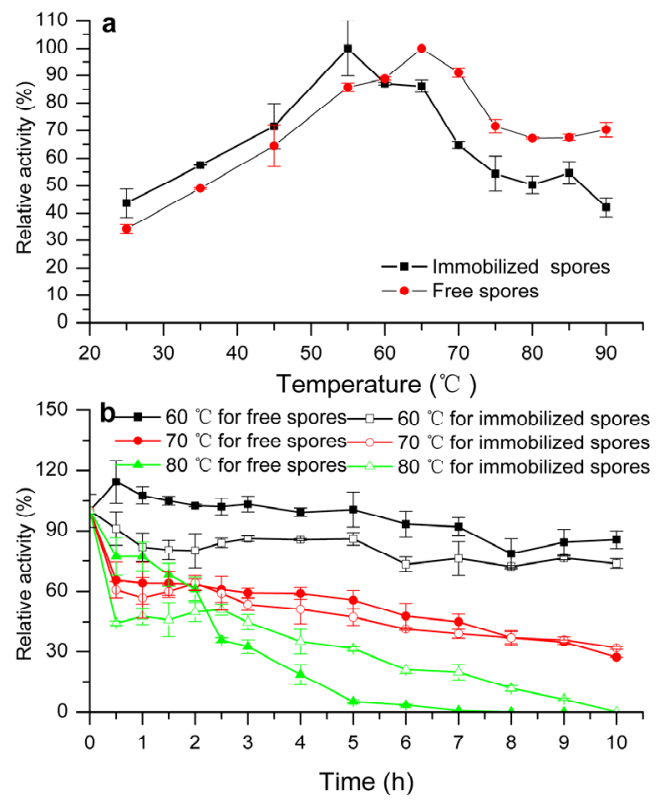

Figure 4: Effect of temperature on free and immobilized spore laccase activity (a) and stability (b) at $\mathrm{pH}$ 6.8. Laccase activity was measured using SGZ as the substrate.

\section{Effect of Organic Solvents on Free and Immobi- lized Spore Laccase Activity}

As shown in Table 1, after $2 \mathrm{~h}$ of incubation at $37^{\circ} \mathrm{C}$ in the presence of $10 \%(\mathrm{v} / \mathrm{v})$ organic solvents, the residual activity of free spore laccase changed a little, except that it was partly inhibited by dimethylsulfoxide and dimethylformamide, while some organic solvents, including acetone, acetonitrile, ethyl acetate and dimethylformamide, promoted the immobilized spore laccase activity greatly. In the presence of $50 \%$ 
$(\mathrm{v} / \mathrm{v})$ organic solvents, the free spore laccase showed low residual activity, between 0 and $48.62 \%$ of its initial activity, while immobilized spore laccase maintained high stability and, specially, it retained more than $80 \%$ of its activity in $50 \%(\mathrm{v} / \mathrm{v})$ methanol or dichloromethane.

Table 1: Effects of organic solvents on the activity of free and immobilized spore laccases.

\begin{tabular}{|c|c|c|c|}
\hline \multirow{2}{*}{$\begin{array}{l}\text { Orgnanic } \\
\text { compounds }\end{array}$} & \multirow{2}{*}{$\begin{array}{c}\text { Concentration } \\
(\%, v / v)\end{array}$} & \multicolumn{2}{|c|}{ Residual activity (\%) } \\
\hline & & $\begin{array}{c}\text { Free } \\
\text { spores }\end{array}$ & $\begin{array}{c}\text { Immobilized } \\
\text { spores }\end{array}$ \\
\hline Control & & $100.00 \pm 0.73$ & $100.00 \pm 1.14$ \\
\hline \multirow[t]{2}{*}{ methanol } & 10 & $110.74 \pm 4.29$ & $99.53 \pm 3.38$ \\
\hline & 50 & $16.68 \pm 1.98$ & $82.54 \pm 2.11$ \\
\hline \multirow[t]{2}{*}{ ethanol } & 10 & $104.2 \pm 3.22$ & $86.2 \pm 5.88$ \\
\hline & 50 & $40.79 \pm 2.56$ & $51.24 \pm 2.23$ \\
\hline \multirow[t]{2}{*}{ acetone } & 10 & $106.12 \pm 4.23$ & $193.87 \pm 2.18$ \\
\hline & 50 & $1.8 \pm 0.23$ & $21.34 \pm 1.08$ \\
\hline \multirow[t]{2}{*}{ acetonitrile } & 10 & $126.09 \pm 4.48$ & $180.78 \pm 6.56$ \\
\hline & 50 & $3.42 \pm 0.33$ & $55.23 \pm 1.27$ \\
\hline \multirow[t]{2}{*}{ Dimethylsulfoxide } & 10 & $84.64 \pm 1.19$ & $108.02 \pm 4.59$ \\
\hline & 50 & $6.78 \pm 0.56$ & $33.55 \pm 1.76$ \\
\hline \multirow[t]{2}{*}{ ethyl acetate ${ }^{a}$} & 10 & $100.84 \pm 5.23$ & $149.41 \pm 6.78$ \\
\hline & 50 & - & + \\
\hline \multirow[t]{2}{*}{ Dichloromethane $^{\mathrm{b}}$} & 10 & $36.47 \pm 3.23$ & $22.05 \pm 1.22$ \\
\hline & 50 & $48.62 \pm 3.12$ & $103.24 \pm 5.59$ \\
\hline \multirow[t]{2}{*}{ Dimethylformamide } & 10 & $79.42 \pm 4.56$ & $128.89 \pm 3.45$ \\
\hline & 50 & $3.54 \pm 0.75$ & $2.33 \pm 0.54$ \\
\hline
\end{tabular}

Note:

a The products generated from laccase oxidizing substrate were in the water phase. It could not be measured in the presence of $50 \%(\mathrm{v} / \mathrm{v})$ ethyl acetate because the reaction mixture was an emulsion. "-" represents no reaction and "+" represents weak reaction.

$\mathrm{b}$ The products generated from laccase oxidizing substrate were in the organic phase. The sample containing dichloromethane was measured with the water phase. The products extracted into the organic phase were not measured (only the sample containning 10\% of diclorornethane has the situation that a small amount of products extracted into the organic phase). In addition, half of its absorbance value was taken as the final value when the samples containing $50 \%$ dichloromethane were measured.

\section{Effect of Metal Ions and Inhibitors on the Free} and Immobilized Spore Laccase Activity

The effects of various metal ions $(10 \mathrm{mM})$ on the free and immobilized spore laccases are listed in Table 2. The results showed that free spore laccase was severely inhibited by $\mathrm{Mn}^{2+}, \mathrm{Fe}^{3+}$ and $\mathrm{Co}^{2+}$, while it was not inhibited by $\mathrm{Na}^{+}, \mathrm{K}^{+}$. Other metal ions had partly inhibitory effects on free spore laccase activity. In contrast, the activity of immobilized spore laccase was improved remarkablely in the solution containing metal ions except it was still severely inhibited by $\mathrm{Mn}^{2+}$ and $\mathrm{Fe}^{3+}$. Some metal ions, such as $\mathrm{Al}^{3+}, \mathrm{K}^{+}, \mathrm{Ca}^{2+}$ and $\mathrm{Zn}^{2+}$ turn from inhibitors into activators after spore immobilization.
Table 2: Effects of metal ions on the activity of free and immobilized spore laccases.

\begin{tabular}{|c|c|c|}
\hline \multirow{2}{*}{$\begin{array}{c}\text { Metal ions } \\
(\mathbf{1 0 ~} \mathbf{~ m M})\end{array}$} & \multicolumn{2}{|c|}{ Residual activity (\%) } \\
\cline { 2 - 3 } & Free spores & Immobilized spores \\
\hline $\mathrm{Control}$ & $100.00 \pm 1.42$ & $100.00 \pm 1.10$ \\
$\mathrm{Na}^{+}$ & $97.22 \pm 2.67$ & $102.67 \pm 3.03$ \\
$\mathrm{Mg}^{2+}$ & $93.09 \pm 4.18$ & $98.78 \pm 6.44$ \\
$\mathrm{Al}^{3+}$ & $91.55 \pm 3.50$ & $111.22 \pm 2.16$ \\
$\mathrm{~K}^{+}$ & $96.52 \pm 2.51$ & $122.78 \pm 6.02$ \\
$\mathrm{Ca}^{2+}$ & $91.32 \pm 1.34$ & $113.56 \pm 5.14$ \\
$\mathrm{Mn}^{2+}$ & $17.48 \pm 1.50$ & $28.44 \pm 2.26$ \\
$\mathrm{Fe}^{3+}$ & $2.07 \pm 0.78$ & $5.00 \pm 1.62$ \\
$\mathrm{Co}^{2+}$ & $30.36 \pm 4.17$ & $92.78 \pm 2.31$ \\
$\mathrm{Ni}^{2+}$ & $75.25 \pm 2.84$ & $96.11 \pm 4.67$ \\
$\mathrm{Cu}^{2+}$ & $79.21 \pm 2.59$ & $95.11 \pm 4.30$ \\
$\mathrm{Zn}^{2+}$ & $78.32 \pm 1.50$ & $110 \pm 6.35$ \\
\hline
\end{tabular}

The effect of several typical laccase inhibitors on the activity of free and immobilized spore laccases is shown in Table 3. The activity of free spore laccase was completely inhibited by $1 \mathrm{mM}$ of cysteine, while it was partially inhibited by $\mathrm{NaN}_{3}$ and PMSF in various concentrations. In contrast, the immobilized spore laccase was found to manifest significant tolerance against inactivation by cysteine, $\mathrm{NaN}_{3}$ and PMSF. Specifically, higher residual activity of immobilized spore laccase was observed in high concentrations of $\mathrm{NaN}_{3}$, while free spore laccase retained its maximum residual activity when the concentration of $\mathrm{NaN}_{3}$ was $0.005 \mathrm{mM}$. Both free and immobilized spore laccases manifest low tolerance towards $1 \mathrm{M}$ of $\mathrm{NaCl}$.

Table 3: Effects of inhibitors on the activity of free and immobilized spore laccases.

\begin{tabular}{|l|r|r|r|}
\hline Inhibitors & Concentration & \multicolumn{2}{|c|}{ Residual activity (\%) } \\
\cline { 3 - 4 } & $(\mathbf{m M )}$ & Free spores & $\begin{array}{r}\text { Immobilized } \\
\text { spores }\end{array}$ \\
\hline Control & & $100.00 \pm 1.22$ & $100.00 \pm 2.87$ \\
\hline EDTA & 10 & $78.18 \pm 4.23$ & $64.69 \pm 4.68$ \\
& 25 & $75.63 \pm 6.14$ & $66.67 \pm 3.74$ \\
& 50 & $59.25 \pm 1.75$ & $51.38 \pm 1.39$ \\
\hline Cysteine & 0.01 & $73.93 \pm 2.35$ & $131.49 \pm 2.87$ \\
& 0.1 & $5.77 \pm 1.22$ & $89.33 \pm 2.01$ \\
& 1 & $0.36 \pm 0.15$ & $71.01 \pm 1.13$ \\
\hline NaN3 & 0.0005 & $68.89 \pm 1.33$ & $37.68 \pm 0.66$ \\
& 0.005 & $79.01 \pm 2.26$ & $85.38 \pm 1.28$ \\
& 0.05 & $51.35 \pm 1.39$ & $98.81 \pm 3.35$ \\
\hline NaCl & 100 & $97.49 \pm 2.22$ & $82.87 \pm 5.59$ \\
& 500 & $99.37 \pm 1.38$ & $89.33 \pm 1.19$ \\
& 1000 & $12.35 \pm 0.51$ & $1.58 \pm 0.33$ \\
\hline SDS & 0.1 & $89.61 \pm 6.88$ & $92.23 \pm 5.29$ \\
& 1 & $50.2 \pm 4.75$ & $81.29 \pm 3.01$ \\
& 10 & $30.25 \pm 3.35$ & $10.14 \pm 1.15$ \\
\hline PMSF & 0.1 & $96.91 \pm 3.33$ & $91.44 \pm 5.43$ \\
& 1 & $101.25 \pm 2.34$ & $91.88 \pm 4.56$ \\
& 10 & $26.44 \pm 0.69$ & $65.22 \pm 1.76$ \\
\hline
\end{tabular}




\section{Dye Decolorization}

\section{Measuring of the Maximum Absorbance Wave- length and Mediator Screening}

The maximum absorbance wavelength of dyes was investigated because it may be influenced by the solvent and $\mathrm{pH}$. The results showed that the maximum adsorption wavelengths of methyl green and acid red 1 were observed at $\lambda=630 \mathrm{~nm}$ and $\lambda=531$ $\mathrm{nm}$, respectively, while the maximum adsorption wavelength of methyl red is $\lambda=520 \mathrm{~nm}$ in acidic conditions and $\lambda=435 \mathrm{~nm}$ in alkaline conditions. In this work, ABTS was demonstrated to be the best mediator compared with SYR, HBT, vanillin and 4-HBA for dye decolorization, and was selected for further study (data not shown).

\section{Effects of Different Decolorization Conditions on Decolorization Efficiency}

In order to find out the characteristics of spore laccase in dye decolorization and to select the suitable condition for further study, the effects of $\mathrm{pH}$, spore amount, dye concentration and treatment time on decolorization efficiency were investigated with free spore laccase (Figure 5). The results indicated that the decolorizaion efficiency of methyl green, methyl red and acid red 1 is the highest at $\mathrm{pH} 7.0, \mathrm{pH}$ 5.0 and pH 6.0, respectively (Figure 5a). Meanwhile, the basic dye (methyl green) decolorized itself severely with the increase in $\mathrm{pH}$ value. The decolorization percentage of all the tested dyes (methyl green, methyl red and acid red 1) increased with the increase of spore amount and reached $86.24 \%, 66.31 \%$ and $65.60 \%$, respectively, for $2.5 \mathrm{mg} / \mathrm{ml}$ of spores (Figure 5b). The highest decolorization efficiency was observed when the concentration of methyl green, methyl red and acid red 1 was $100 \mathrm{mg} / \mathrm{L}, 50$ $\mathrm{mg} / \mathrm{L}$ and $50 \mathrm{mg} / \mathrm{L}$, respectively (Figure $5 \mathrm{c}$ ). The effects of treatment time on the decolorization efficiency varied due to dye differences (Figure 5d). The decolorization percentage of the dyes rose dramatically as the treatment time increased from 0 to $24 \mathrm{~h}$. The decolorization percentages of methyl green and acid red 1 increase slowly when the treatment time exceeded $24 \mathrm{~h}$.

Considering the cost of spore production, decolorization efficiency and the requirements of textile wastewater treatment, a decolorization condition was selected for further study, which was $\mathrm{pH} 7.0,2.5$ $\mathrm{mg} / \mathrm{mL}$ of spore, $100 \mathrm{mg} / \mathrm{L}$ of methyl green or $50 \mathrm{mg} / \mathrm{L}$ of methyl red or acid red 1 , and $24 \mathrm{~h}$ of treatment time.
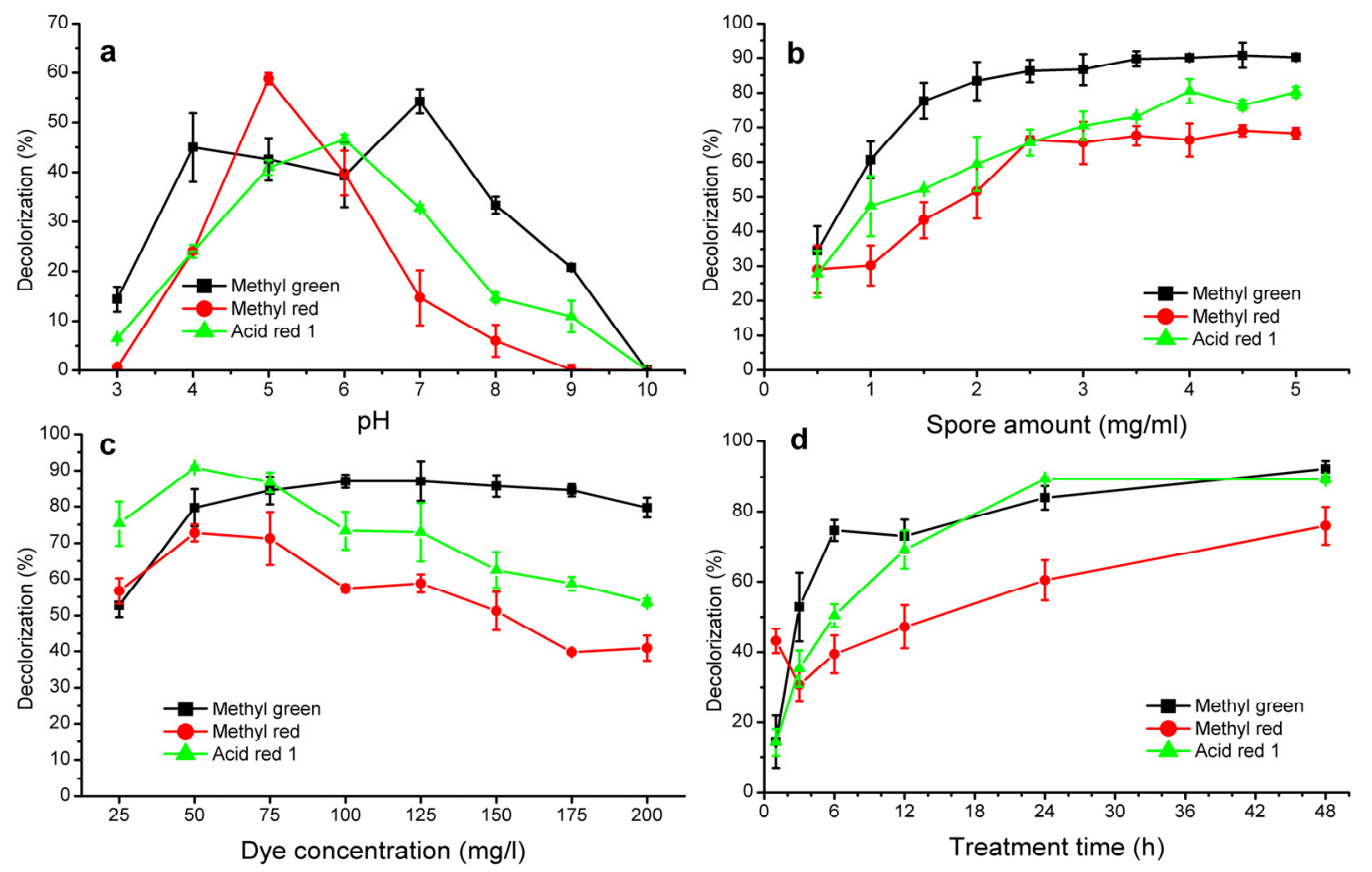

Figure 5: Effect of $\mathrm{pH}(\mathrm{a})$, spore amount (b), dye concentration (c) and treatment time (d) on decolorization efficiency. (a) The reaction mixture $(4 \mathrm{ml})$ contained $1 \mathrm{mM} \mathrm{ABTS}, 100 \mathrm{mg} / \mathrm{L}$ dyes and $1.0 \mathrm{mg} / \mathrm{mL}$ free spores. The samples were incubated at $37^{\circ} \mathrm{C}, \mathrm{pH} 3.0-10.0$ and rotated at $200 \mathrm{rpm}$ for $24 \mathrm{~h}$. Subsequent experimental conditions were set according to the optimum conditions from the previous experiment: (b) $\mathrm{pH} 7.0$; (c) 2.5 $\mathrm{mg} / \mathrm{mL}$ spores; (d) methyl green (100 mg/L), methyl red (50 mg/L) and acid red 1 (50 mg/L). 
Dye Decolorization by Free and Immobilized Spore Laccases

The decolorization ability of free and immobilized spore laccases was evaluated with two acid dyes (methyl red and acid red 1) and a basic dye (methyl green) at $37{ }^{\circ} \mathrm{C}$ and $60{ }^{\circ} \mathrm{C}$ at $\mathrm{pH}$ 7.0. As shown in Figure 6, methyl green, methyl red and acid red 1 were removed $86.82 \%, 78.14 \%$ and $88.60 \%$, respectively, by immobilized spore laccase, while they were removed $76.61 \%, 59.03 \%$ and $81.03 \%$, respectively, by free spore laccase at $37{ }^{\circ} \mathrm{C}$. In addition, the DEAE-cellulose without spores had high adsorption ability towards acid dyes (methyl red and acid red 1). It absorbed $42.49 \%$ of methyl red and $77.19 \%$ of acid red 1, while methyl green was decolorized $6.85 \%$ at $37{ }^{\circ} \mathrm{C}$. There was no significant difference of the decolorization efficiency whether the reaction mixture was incubated at $37^{\circ} \mathrm{C}$ or $60{ }^{\circ} \mathrm{C}$.

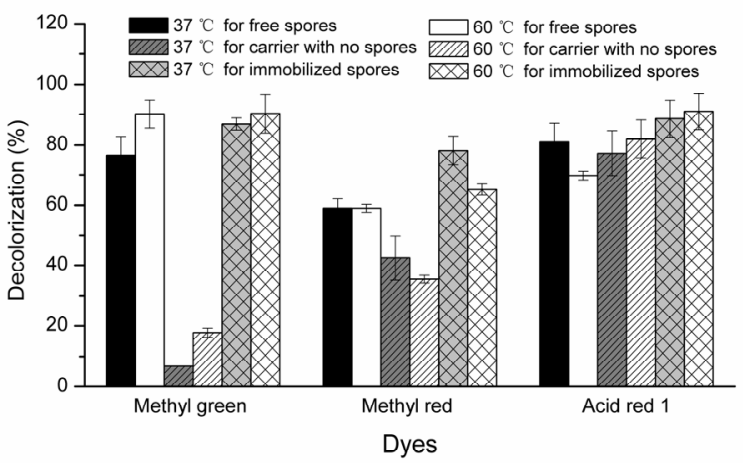

Figure 6: Decolorization of synthetic dyes by free and immobilized spore laccases. Each reaction sample contained $10 \mathrm{mg}$ spores or immobilized spores (does not include the DEAE-cellulose weight). Their decolorization ability was evaluated by measuring the residual absorbance of methyl green $(100 \mathrm{mg} / \mathrm{L})$, methyl red $(50 \mathrm{mg} / \mathrm{L})$ and acid red $1(50 \mathrm{mg} / \mathrm{L})$ after $24 \mathrm{~h}$ incubation at $37^{\circ} \mathrm{C}, \mathrm{pH} 7.0$ and $200 \mathrm{rpm}$.

\section{The Reusability of Immobilized Spore Laccase}

In order to evaluate the reusability of immobilized spore laccase, the decolorization percentages of all the tested dyes were determined after multiple reusage as shown in Figure 7. The immobilized spore laccase retained more than $95 \%$ of its initial activity in the 2nd cycle of dye decolorization. With increasing repeat cycles, the immobilized spore laccase showed a different reusability for the three dyes. The immobilized spore laccase retained $51.03 \%$, $62.56 \%$ and $74.34 \%$ of its initial decolorization ac- tivity for decolorizing methyl green, methyl red and acid red 1 after 7 cycles of reusage.

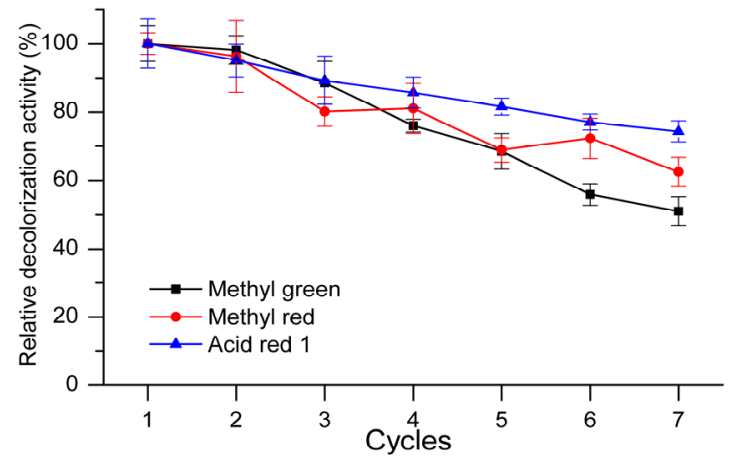

Figure 7: Reusability of immobilized spore laccase for dye decolorization.

\section{DISCUSSION}

DEAE-cellulose is a positively charged resin. In ion exchange adsorption, the adsorption $\mathrm{pH}$ is the most important parameter to determine which object is adsorbed (Nakatani et al., 2012). The optimum $\mathrm{pH}$ of spore laccase for immobilization $(\mathrm{pH} 10.0$, Figure 1) is different from the immobilization of puerarin glycosidase on DEAE-52 cellulose, where the adsorption on DEAE-52 cellulose decreased when the $\mathrm{pH}$ was higher or lower than 6.5 (Liu et al., 2014). The optimum $\mathrm{pH}$ of immobilization is related to the isoelectric point of the protein and its resistance towards acid and alkaline conditions. When an anion exchanger is used as the immobilization carrier, the higher the isoelectric point, the higher the adsorption $\mathrm{pH}$ condition of immobilization. Besides, the more alkali-resistant the enzyme, the broader the acceptable $\mathrm{pH}$ range of immobilization. The properties of alkali-resistance of spore laccase contribute to getting a more stable immobilized spore laccase through ionic bonding, because a $\mathrm{pH}$ far away from the isoelectric point of the molecule of interest will give stronger binding and increased capacity (Amersham Pharmacia Biotech, 1999). However, the chemical structure of puerarin glycosidase might be destroyed under alkaline conditions, which made the activity of immobilized puerarin glycosidase decrease fast when the $\mathrm{pH}$ was higher than 6.5 (Liu et al., 2014).

The current results are similar to the immobilization of nuclease $\mathrm{p} 1$ on DEAE-cellulose (Shi et al., 2010). DEAE-cellulose can absorb a lot of spores in a short time, but the spore distribution on the fiber surface is not uniform (Figure 2). Those fibers whose surface is crinkly or dehiscent coupled a large number of spores, while most fibers that have a smooth 
surface often coupled less spores. This may be related to the contact area between spores and fibers, steric hindrance and the distribution of di-ethyl amino-ethyl tertiary amine functional groups on the surface of the fibers. The inner spores were affected by diffusion constraints when a large number of spores were stacked together on the surface of fibers, so that the laccase activity of immobilized spores did not increase with increasing spore amount (Figure 2c) (Cao 2006). The dry immobilized spore laccase with a relief-like morphology may generate stronger binding between spores and fibers, but it could also affect the enzyme activity adversely (Figure $2 \mathrm{f}$ ).

The $\mathrm{pH}$ preferences of spores after immobilization show no significant changes (Figure 3a). The optimum $\mathrm{pH}$ of free and immobilized spore laccases for oxidation of ABTS is different from most Bacillus laccases (Lončar et al., 2013; Guan et al., 2014b; Lu et al., 2013) where their optimum $\mathrm{pH}$ for laccase activity was between $\mathrm{pH} 3.8$ and $\mathrm{pH} 4.8$. The optimum $\mathrm{pH}$ of free and immobilized spore laccases for oxidation of SGZ and 2, 6-DMP corresponds to that of other Bacillus laccases (Wang et al., 2011; Koschorreck et al., 2008) in the $\mathrm{pH}$ range of 6.5-7.5 (Zhou et al., 2015). However, fungal laccase has lower $\mathrm{pH}$ preference than spore laccase. They usually oxidize ABTS in the $\mathrm{pH}$ range of 2.0-4.0 and oxidize SGZ and DMP in the $\mathrm{pH}$ range of 3.0-6.2 (Baldrian 2006). The $\mathrm{pH}$ stability of free and immobilized spore laccase from B. pumilus W3 is similar to that of laccase from Bacillus vallismortis fmb-103 (Zhang et al., 2012; Zhang et al., 2013), and stronger than that of spore laccase from Bacillus SF (Held et al. 2005). In contrast, fungal laccases are extremely unstable under alkaline conditions, for instance, a laccase from Trametes versicolor was inactivated completely after $1 \mathrm{~h}$ at $60{ }^{\circ} \mathrm{C}$ at $\mathrm{pH} 7.0$ or $\mathrm{pH} 8.0$ (Carvalho et al., 1999). Moreover, the $\mathrm{pH}$ stability of spore laccase after immobilization was improved significantly (Figure 3b). These results showed the advantages of immobilized spore laccase applied under alkaline conditions.

The optimum temperature of free and immobilized spore laccase (Figure 4a) is similar to that of some Bacillus laccases such as spore laccase from $B$. licheniformis LS04 (Lu et al., 2012a). According to a previous report (Zhou et al., 2015), the optimum reaction temperature of laccase from Bacillus strains is usually between $60{ }^{\circ} \mathrm{C}$ and $80^{\circ} \mathrm{C}$, while the optimum temperature of fungal laccase is usually between $50^{\circ} \mathrm{C}$ and $70{ }^{\circ} \mathrm{C}$ (Baldrian 2006). Bacillus laccase is more thermostable than fungal laccase, even though there is little difference between their optimum temperatures. In the report by Hiden et al.
(2009), the half-life of fungal laccase was usually not more than $1 \mathrm{~h}$, while in this study the half-lives of free and immobilized spore laccase were more than 4 $\mathrm{h}$ at $70{ }^{\circ} \mathrm{C}$. The results (Figure $4 \mathrm{~b}$ ) also indicated that free laccase can retain higher activity at short times and lower temperatures, while immobilized spore laccase has the advantage at long times and high temperature.

The effects of several organic solvents such as methanol, ethanol, etc. on the laccase activity of free and immobilized spores are very different. Spore laccase after immobilization has obvious advantages in organic solvents (Table 1) may relate to the fact that free and immobilized spore laccases have different preferences towards water activity and aquaphilicity. According to a previous report (Cao, 2008), different carriers and protein amount influence the activity of immobilized enzymes because they may change the water activity of the solution. The effects of metal ions on the activity of free and immobilized spore laccase from B. pumilus W3 (Table 2) are similar to that of other Bacillus laccases whose activity is usually activated by $\mathrm{Li}^{+}, \mathrm{Na}^{+}, \mathrm{Mg}^{2+}, \mathrm{Ca}^{2+}$ and $\mathrm{Ba}^{2+}$, and inhibited by $\mathrm{Ag}^{+}, \mathrm{Hg}^{2+}$ and $\mathrm{Fe}^{3+}$ (Zhou et al., 2015). The spore laccase after immobilization improved its stability towards metal ions, which may be related to the change of structure of the laccase active center when the spores are immobilized. A similar situation can also be found in a previous report (Asghe et al., 2012). However, the effects of metal ions on the catalytic activity of enzymes are very complex and there are no one-to-one relationships between individual metals and functions (Andreini et al. 2008). So, the mechanism of metal ions effects on spore laccase should be studied further. The free spore laccase was stability towards some typical laccase inhibitors similar to other Bacillus laccases (Zhou et al., 2015) and its activity was inhibited to varying degrees (Table 3 ). Specially, similar to a previous report (Zhang et al., 2012), it was strongly inhibited by cysteine, $\mathrm{NaN}_{3}$ and PMSF. In contrast, the immobilized spore laccase manifested strong resistance towards high concentrations of cysteine, $\mathrm{NaN}_{3}$ and PMSF. What caused this difference is unclear and needs to be studied further.

One of the most important factors that influences the decolorization efficiency is $\mathrm{pH}$. The optimum $\mathrm{pH}$ $(\mathrm{pH}$ 5.0-7.0) for decolorizaiton of methyl green, methyl red and acid red 1 by spore laccase from $B$. pumilus W3 (Figure 5a) is higher than that of the spore laccase from $B$. vallismortis, which has high decolorization efficiency in the $\mathrm{pH}$ range of 4.0-6.0, and that of the laccase from Trametes modesta, which has no decolorization activity when the $\mathrm{pH}$ 
value is higher than 7 (Held et al. 2005; Zhang et al., 2012). However, the $\mathrm{pH}$ of combined effluents from textile industries is usually in the $\mathrm{pH}$ range of 6.0 11.0 (Yu et al., 2013). This indicates the potential of spore laccase from B. pumilus W3 in textile dye wastewater treatment. Similar to some other spore laccases (Lu et al., 2012a; Lončar et al., 2013), the spore laccase from B. pumilus W3 has high decolorization ability when the dye concentration is between 25-200 mg/l (Figure 5c). Considering the time and decolorization efficiency, $24 \mathrm{~h}$ of treatment time is suitable for spore laccase in dye decolorization (Figure 5d). Some Bacillus species cultures can also degrade synthetic dyes with high efficiency, but usually need a longer time than spore laccase (Dawkar et al., 2009; Kadam et al., 2013).

Both free and immobilized spore laccases had high decolorization ability towards three dyes (Figure $6)$. The lowest decolorization efficiency calculated for free spore laccase oxidizing methyl red was still $58.96 \%$. Immobilized spore laccase with the same weight of spores as free spore laccase showed a higher decolorizing percentage for all the tested dyes compared to free spore laccase. Similar results were obtained by Mirzadeh et al., (2014), where the immobilized laccase had higher decolorization efficiency than free laccase. DEAE-cellulose can adsorb some dyes and its adsorption efficiency may be decided by its charge. Specially, acid red 1 could be efficiently absorbed by DEAE-cellulose and be decolorized $81.95 \%$ at $60{ }^{\circ} \mathrm{C}$. This situation showed an advantage of immobilized spore laccase since the adsorption of carrier towards dyes can promote the decolorization efficiency of dyes, and can also decolorize some dyes that can not be decolorized by spore laccase.

The immobilized spore laccase can be reused for at least 7 cycles in dye decolorization (Figure 7). This is much higher than the reusage times of immobilized laccase on a similar carrier, green coconut fiber, where immobilized laccase lost $40 \%-100 \%$ of its decolorization activity towards different dyes after 3 reusages (Cristóvão et al., 2012). In the previous report, spore laccase immobilized in calcium alginate beads showed a higher reusability than this study and could be reused at least 15 times (Lu et al., 2012b). However, calcium alginate beads usually exhibit a lot of water uptake and subsequently dissolve in phosphate buffer under alkaline conditions (Bajpai and sharma, 2004). The dissolution is caused by the ion-exchange between $\mathrm{Ca}^{2+}$ and $\mathrm{Na}^{+}$in phosphate buffered saline, and may also be caused by the ion-exchange between $\mathrm{Ca}^{2+}$ and other metal ions in other solutions. This limits the application of immo- bilized spore laccase in calcium alginate. In contrast, considering that the loss of activity in multi-cycle reusage may be caused by desorption of spores, adding spores to re-immobilize after 7 cycles of reusage may improve the reusage times of immobilized spore laccase greatly and could finish with a simple adsorption.

In conclusion, on the basis of previous reports, this study presents detailed research on the immobilization and characterization of spore laccase and its application in dye decolorization (Table A1). The immobilized spore laccase from B. pumilus $\mathrm{W} 3$ was prepared by a simple method and from cheap materials, has high stability towards various harsh environments, and can decolorize various dyes efficiently and be reused many times. These properties demonstrate that immobilized spore laccase might be useful for textile effluent treatment.

\section{ACKNOWLEDGEMENTS}

This work was supported by the National Natural Science Foundation of China (31472003 \& 31101331), the Open Project Program of the Key Laboratory of Industrial Biotechnology, Ministry of Education, China (KLIB-KF201304), a Project Funded by the Priority Academic Program Development of Jiangsu Higher Education Institutions, the 111 Project (No. 111-2-06), and the Jiangsu province "Collaborative Innovation Center for Advanced Industrial Fermentation" industry development program. We thank Julie from Wuxi Joy English School for language assistance.

\section{REFERENCES}

Amersham Pharmacia Biotech., Ion Exchange Chromatography: Principles and Methods. Amersham Pharmacia Biotech. Stockholm. (1999).

Andreini, C., Bertini, I., Cavallaro, G., Holliday, G. L., Thomton, J. M., Metal ions in biological catalysis: from enzyme databases to general principles. Journal of Biological Inorganic Chemistry, 13, 1205-1218 (2008).

Asghe, M., Iqbal, H. M. N., Irshad, M., Characterization of purified and Xerogel immobilized Novel Lignin Peroxidase produced from Trametes versicolor IBL-04 using solid state medium of Corncobs. BMC Biotechnology, 12, 46 (2012).

Bajpai, S. K., Sharma, S., Investigation of swelling/degradation behaviour of alginate beads crosslinked with $\mathrm{Ca}^{2+}$ and $\mathrm{Ba}^{2+}$ ions. Reactive and 
Functional Polymers, 59, 129-140 (2004).

Baldrian, P., Fungal laccases - occurrence and properties. FEMS Microbiology Letters, 30, 215-242 (2006).

Bayramoglu, G., Gursel, I., Yilmaz, M., Arica, M. Y., Immobilization of laccase on itaconic acid grafted and $\mathrm{Cu}(\mathrm{II})$ ion chelated chitosan membrane for bioremediation of hazardous materials. Journal of Chemical Technology and Biotechnology, 87, 530-539 (2012).

Cao, L., Carrier-Bound Immobilized Enzymes: Principles, Application and Design. Wiley-VCH Verlag GmbH \& Co. KGaA. Weinheim. doi:10.1002/ 3527607668.ch1 (2006).

Cristóvão, R. O., Silvério, S. C., Tavares, A. P. M., Brígida, A. I. S., Loureiro, J. M., Boaventura, R. A. R., Macedo, E. A., Coelho, M. A. Z., Green coconut fiber: a novel carrier for the immobilization of commercial laccase by covalent attachment for textile dyes decolourization. World Journal of Microbiology and Biotechnology, 28, 2827-2838 (2012).

da Silva, A. M., Tavares, A. P. M., Rocha, C. M. R., Cristóvão, R. O., Teixeira, J. A., Macedo, E. A., Immobilization of commercial laccase on spent grain. Process Biochemistry, 47, 1095-1101 (2012).

Dawkar, V. V., Jadhav, U. U., Ghodake, G. S., Govindwar, S. P., Effect of inducers on the decolorization and biodegradation of textile azo dye Navy blue 2 GL by Bacillus sp. VUS. Biodegradation, 20, 777-787 (2009).

de Carvalho, M. E. A., Monteiro, M. C., Sant'Anna, G. L., Laccase from Trametes versicolor. Twentieth Symposium on Biotechnology for Fuels and Chemicals, Humana Press, 723-733 (1999).

Fernández-Fernández, M., Sanroman, M. Á., Moldes, D., Recent developments and applications of immobilized laccase. Biotechnology Advance, 31, 1808-1825 (2013).

Guan, Z. B., Song, C. M., Zhang, N., Zhou, W., Xu, C. W., Zhou, L. X., Zhao, H., Cai, Y. J., Liao, X. R., Overexpression, characterization, and dyedecolorizing ability of a thermostable, $\mathrm{pH}$-stable, and organic solvent-tolerant laccase from Bacillus pumilus W3. Journal of Molecular Catalysis, B, Enzymatic, 101, 1-6 (2014a)

Guan, Z. B., Zhang, N., Song, C. M., Zhou, W., Zhou, L. X., Zhao, H., Xu, C. W., Cai, Y. J., Liao, X. R., Molecular cloning, characterization, and dye-decolorizing ability of a temperature- and pH-stable laccase from Bacillus subtilis X1. Applied Biochemistry and Biotechnology, 172, 1147-1157 (2014b).
Held, C., Kandelbauer, A., Schroeder, M., CavacoPaulo, A., Guebitz, G. M., Biotransformation of phenolics with laccase containing bacterial spores. Environmental Chemistry Letters, 3, 74-77 (2005).

Hilden, K., Hakala, T. K., Lundell, T., Thermotolerant and thermostable laccases. Biotechnology Letters, 31, 1117-1128 (2009).

Jenkinson, H. F., Sawyer, W. D., Mandelstam, J., Synthesis and order of assembly of spore coat proteins in Bacillus subtilis. Journal of General Microbiology, 123, 1-16 (1981).

Kadam, A. A., Kamatkar, J. D., Khandare, R. V., Jadhav, J. P., Govindwar, S. P., Solid-state fermentation: Tool for bioremediation of adsorbed textile dyestuff on distillery industry waste-yeast biomass using isolated Bacillus cereus strain EBT1. Environmental Science and Pollution Research, 20, 1009-1020 (2013).

Koschorreck, K., Richter, S. M., Ene, A. B., Roduner, E., Schmid, R. D., Urlacher, V. B., Cloning and characterization of a new laccase from Bacillus licheniformis catalyzing dimerization of phenolic acids. Applied Microbiology and Biotechnology, 79, 217-224 (2008).

Liu, G. Y., Sun, L., Wu, X. X., Zhang, W., Feng, J. S., Cui, Y., Lu, Z., Shen, J. J., Liu, Z. H., Yuan, S., Immobilization of puerarin glycosidase from Microbacterium oxydans CGMCC 1788 increases puerarin transformation efficiency. Brazilian Journal of Chemical Engineering, 31, 325-333 (2014).

Lončar, N., Božić, N., Lopez-Santin, J., Vujčić, Z., Bacillus amyloliquefaciens laccase - from soil bacteria to recombinant enzyme for wastewater decolorization. Bioresource Technology, 147, 177-183 (2013).

Lončar, N., Gligorijević, N., Božić, N., Vujčić, Z., Congo red degrading laccases from Bacillus amyloliquefaciens strains isolated from salt spring in Serbia. International Biodeterioration \& Biodegradation, 91, 18-23 (2014).

Lu, L., Wang, T. N., Xu, T. F., Wang, J. Y., Wang, C. L., Zhao, M., Cloning and expression of thermoalkali-stable laccase of Bacillus licheniformis in Pichia pastoris and its characterization. Bioresource Technology, 134, 81-86 (2013).

Lu, L., Zhao, M., Li, G. F., Li, J., Wang, T. N., Li, D. B., Xu, T. F., Decolorization of synthetic dyes by immobilized spore from Bacillus amyloliquefaciens. Catalysis Communications, 26, 58-62 (2012b).

Lu, L., Zhao, M., Wang, T. N., Zhao, L. Y., Du, M. H., Li, T. L., Li, D. B., Characterization and dye decolorization ability of an alkaline resistant and 
organic solvents tolerant laccase from Bacillus licheniformis LS04. Bioresource Technology, 115, 35-40 (2012a).

Madhavi, V., Lele, S. S., Laccase: Properties and applications. Bioresources, 4, 1694-1717 (2009).

Mirzadeh, S. S., Khezri, S. M., Rezaei, S., Forootanfar, H., Mahvi, A. H., Faramarzi, M. A., Decolorization of two synthetic dyes using the purified laccase of Paraconiothyrium variabile immobilized on porous silica beads. Journal of Environmental Health Science and Engineering, 12, 6 (2014).

Mogharabi, M., Nassiri-Koopaei, N., Bozorgi-Koushalshahi, M., Nafissi-Varcheh, N., Bagherzadeh, G.., Faramarzi, M. A., Immobilization of laccase in alginate-gelatin mixed gel and decolorization of synthetic dyes. Bioinorganic Chemistry and Applications (2012). doi: 10.1155/2012/823830

Nakatani, N., Kozaki, D., Mori, M., Tanaka, K., Recent progress and applications of ion-exclusion/ ion-exchange chromatography for simultaneous determination of inorganic anions and cations. Analytical Sciences, 28, 845-852 (2012).

Ozmen, E. Y., Sezgin, M., Yilmaz, A., Yilmaz, M., Synthesis of $\beta$-cyclodextrin and starch based polymers for sorption of azo dyes from aqueous solutions. Bioresource Technology, 99, 526-531 (2008).

Sarayu, K., Sandhya, S., Current technologies for biological treatment of textile wastewater-a review. Applied Biochemistry and Biotechnology, 167, 645-661 (2012).

Schaeffer, P., Millet, J., Aubert J. P., Catabolic repression of bacterial sporulation. Proceedings of the National Academy of Sciences of the United States of America, 54, 704-711 (1965).

Shi, L. E., Yi, Y., Tang, Z. X., Xiong, W. Y., Mei, J. F., Ying, G. Q., Nuclease p1 immobilization of DEAE cellulose. Brazilian Journal of Chemical Engineering, 27, 31-39 (2010).

Strong, P. J., Claus, H., Laccase: A review of its past and its future in bioremediation. Critical Reviews in Environmental Science and Technology, 41, 373-434 (2011).

Valerio, S. G., Alves, J. S., Klein, M. P., Rodrigues,
R. C., Hertz, P. F., High operational stability of invertase from Saccharomyces cerevisiae immobilized on chitosan nanoparticles. Carbohydrate Polymers, 92, 462-468 (2013).

van Waasbergen, L. G., Hildebrand, M., Tebo, B. M., Identification and characterization of a gene cluster involved in manganese oxidation by spores of the marine Bacillus sp. strain SG-1. Journal of Bacteriology, 178, 3517-3530 (1996).

Wang, C. L., Zhao, M., Lu, L., Wei, X. D., Li, T. L., Characterization of spore laccase from Bacillus subtilis WD23 and its use in dye decolorization. African Journal of Biotechnology, 10, 2186-2192 (2011).

Wang, H. X., Zhang, W., Zhao, J. X., Xu, L. L., Zhou, C. Y., Chang, L., Wang, L. Y., Rapid decolorization of phenolic azo dyes by immobilized laccase with $\mathrm{Fe}_{3} \mathrm{O}_{4} / \mathrm{SiO}_{2}$ nanoparticles as support. Industrial \& Engineering Chemistry Research, 52, 4401-4407 (2013).

Wesenberg, D., Kyriakides, I., Agathos, S. N., White-rot fungi and their enzymes for the treatment of industrial dye effluents. Biotechnology Advances, 22, 161-187 (2003).

Yu, G. S., Textile Printing and Dyeing Wastewater Treatment and Recycling. Industrial Wastewater Treatment and Regeneration Utilization. Yu, G. S., Guo, M. X., Huang, J. Y., Eds., Chemical Industry Press. Beijing, 132-183 (2013). (In Chinese)

Zhang, C., Diao, H. W., Lu, F. X., Bie, X. M., Wang, Y. F., Lu, Z. X., Degradation of triphenylmethane dyes using a temperature and $\mathrm{pH}$ stable spore laccase from a novel strain of Bacillus vallismortis. Bioresource Technology, 126, 80-86 (2012).

Zhang, C., Zhang, S., Diao, H. W., Zhao, H. Z., Zhu, X. Y., Lu, F. X., Lu, Z. X., Purification and characterization of a temperature- and $\mathrm{pH}$-stable laccase from the spores of Bacillus vallismortis fmb-103 and its application in the degradation of malachite green. Journal of Agricultural and Food Chemistry, 61, 5468-5473 (2013).

Zhou, W., Guan, Z. B., Cai, Y. J., Song, C. M., Liao, X. R., Progress in the Bacillus laccase. Microbiology China, 42, 1372-1383 (2015). (In Chinese). 